\title{
$i^{\rightarrow+3}$
}

NASA Contractor Report 191595

ICASE Report No. 94-5

$1 N-64$

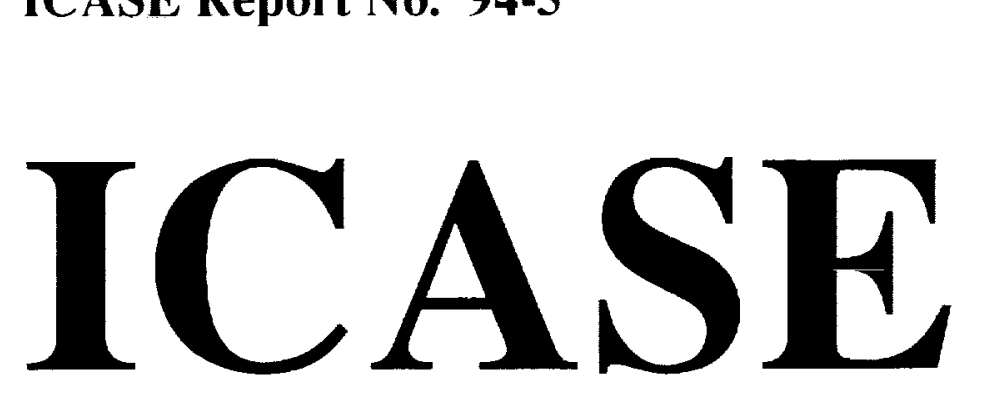

314 $p^{29}$

\section{AGGLOMERATION MULTIGRID FOR THE THREE-DIMENSIONAL EULER EQUATIONS}

\section{Venkatakrishnan}

\section{J. Mavriplis}

NASA Contract No. NAS 1-19480

January 1994

Institute for Computer Applications in Science and Engineering NASA Langley Research Center Hampton, Virginia 23681-0001

Operated by the Universities Space Research Association

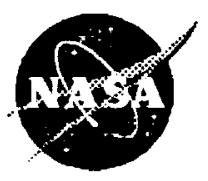

National Aeronautics and Space Administration 


\title{
AGGLOMERATION MULTIGRID FOR THE THREE-DIMENSIONAL EULER EQUATIONS
}

\author{
V. Venkatakrishnan * D. J. Mavriplis ${ }^{\dagger}$ \\ Institute for Computer Applications in Science and Engineering \\ MS 132C, NASA Langley Research Center \\ Hampton, VA 23681-0001
}

\begin{abstract}
A multigrid procedure that makes use of coarse grids generated by the agglomeration of control volumes is advocated as a practical approach for solving the threedimensional Euler equations on unstructured grids about complex configurations. It is shown that the agglomeration procedure can be tailored to achieve certain coarse grid properties such as the sizes of the coarse grids and aspect ratios of the coarse grid cells. The agglomeration is done as a preprocessing step and runs in linear time. The implications for multigrid of using arbitrary polyhedral coarse grids are discussed. The agglomeration multigrid technique compares very favorably with existing multigrid procedures both in terms of convergence rates and elapsed times. The main advantage of the present approach is the ease with which coarse grids of any desired degree of coarseness may be generated in three dimensions, without being constrained by considerations of geometry. Inviscid flows over a variety of complex configurations are computed using the agglomeration multigrid strategy.
\end{abstract}

"Part of this work was done while the author was employed by Computer Sciences Corporation, Moffett Field, CA during which time the work was supported by the National Aeronautics and Space Administration under the NASA contract NAS 2-12961. This research was also supported under the NASA contract No. NAS1-19480 while the author was in residence at the Institute for Computer Applications in Science and Engineering (ICASE), NASA Langley Research Center, Hampton, VA 23681.

${ }^{\dagger}$ This research was supported by the National Aeronautics and Space Administration under the NASA contract No. NAS1-19480 while the author was in residence at the Institute for Computer Applications in Science and Engineering (ICASE), NASA Langley Research Center, Hampton, VA 23681. 


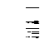




\section{Introduction}

Over the last few years, the multigrid method has been demonstrated as an efficient means for obtaining steady-state solutions to the Euler equations on unstructured meshes in two and three dimensions [1-9]. The main difficulty with multigrid techniques on unstructured meshes is the generation of coarse grids. In the case of structured grids, coarse grids are easily derived from a given fine grid by omitting alternate grid lines in each coordinate direction. In the case of unstructured grids, three different approaches can be adopted.

The first approach begins with a coarse mesh definition and generates finer grids by uniform refinement $[5,6]$. The advantage is that the inter-grid operators become simple because of the nesting of grids. There are several disadvantages with this approach. The first drawback is the dependence of the fine grid point distribution on the coarse levels. Ideally, the fine grid points should be distributed to capture features of the geometry and to control the aspect ratios of the grid cells and grid stretching, whereas in this method, the coarsest grid determines these features. Furthermore, the coarsest level that can be used in the multigrid is predetermined. This may be too fine if the geometry is to be captured on this coarse grid.

The second approach uses non-nested unstructured grids as coarse grids and has been shown to be quite successful in both two- and three-dimensional unstructured grid computations $[1,2,3,4]$. Grids of varying degrees of coarseness are generated independently using any given grid generation strategy. Piecewise-linear interpolation operators for the transference of flow variables, residuals and correction are derived during a preprocessing step by using efficient search procedures. Since the coarse grids are generated independently from one another, the coarse grids are not nested with the fine grids and, in general, do not even contain points in common with the fine levels. A variant of this approach chooses a subset of the fine grid points and retriangulates them [7]. The grids are still non-nested even though the coarse grid points are contained in the fine grid.

The approaches outlined above share a common problem, that of generating coarse grids. For complex geometries, especially in three dimensions, constructing coarse grids that faithfully represent the complex geometries can become a difficult proposition. In other words, there comes a point when the critical features of geometry are too fine to be captured by the coarse grid, with the result that the geometry or sometimes even the topology is altered. The requirement to generate not just one grid but multiple grids that preserve the geometry places too much of a burden on a user. One approach that circumvents this problem is the generation of coarse grids through agglomeration as developed by Lallemand et al. [8] and independently by Smith [9]. Lallemand et al. use a base scheme that stores the variables at the vertices of the triangular mesh, whereas Smith uses a scheme that stores the variables at the centers of triangles. In the present work, a vertex-based scheme is employed. Twodimensional triangular grids contain twice as many cells as vertices (neglecting boundary effects), and in practice, three-dimensional tetrahedral meshes contain 5 to 6 times more cells than vertices. Thus, on a given grid, a vertex scheme incurs substantially less computational overhead than a cell-based scheme. Increased accuracy may be expected from a cell-based scheme, since this involves the solution of a larger number of unknowns. However, the increase in accuracy does not appear to justify the additional computational overheads, particularly in three dimensions. 
The central idea behind the agglomeration strategy of Lallemand et al. [8] is to fuse or agglomerate the control volumes for the vertices using heuristics. The centroidal dual, composed of segments of the median planes of the triangulation, is a collection of the control volumes over which the Euler equations in integral form are solved. For simple geometries and on relatively coarse grids, Lallemand et al. were able to show that the agglomerated multigrid technique performed as well as the multigrid technique which makes use of non-nested coarse grids. However, the convergence rates, especially for the second order accurate version of the scheme, appeared to degrade somewhat. Furthermore, the validation of such a strategy for more complicated geometries and using much finer grids, as well as the incorporation of viscous terms for the Navier-Stokes equations remains to be demonstrated. The work of Smith [9] constitutes the basis of a commercially available computational fluid dynamics code, and as such has been applied to a number of complex geometries [10]. However, consistently competitive multigrid convergence rates have yet to be demonstrated.

In the present work, the agglomeration multigrid strategy is explored further. The issues involved in a proper agglomeration and the implications for the choice of the restriction and prolongation operators are addressed. Inviscid flows over non-simple two and threedimensional geometries involving a large number of unknowns are solved with the agglomeration multigrid strategy. This approach is compared with the unstructured multigrid algorithm of $[1,2]$ which makes use of non-nested coarse grids. Convergence rates as well as CPU times on a Cray Y-MP/1 are compared using both methods.

\section{Governing equations and discretization}

The Euler equations in integral form for a control volume $\Omega$ with boundary $\partial \Omega$ read

$$
\frac{d}{d t} \int_{\mathbf{\Omega}} \boldsymbol{u} d v+\oint_{\partial \boldsymbol{\Omega}} \boldsymbol{F}(\boldsymbol{u}, \boldsymbol{n}) d S=0 .
$$

Here $u$ is the solution vector comprised of the conservative variables: density, the components of momentum, and total energy. The vector $F(u, n)$ represents the inviscid flux vector for a surface with normal vector $n$. Equation (1) states that the time rate of change of the variables inside the control volume is the negative of the net flux of the variables through the boundaries of the control volume. This net flux through the control volume boundary is termed the residual. In the present scheme, the variables are stored at the vertices of a mesh composed of simplices i.e., triangles in two dimensions and tetrahedra in three dimensions. Most of the description in the paper will deal with the three-dimensional situation, the reduction to the two-dimensional situation being obvious. The control volumes are nonoverlapping polyhedra which surround the vertices of the mesh. They form the dual of the mesh, which is composed of segments of median planes. Associated with each edge of the original mesh is a (segmented) dual face. The contour integrals in Equation (1) are replaced by discrete path integrals over the faces of the control volume which are computed by using the trapezoidal rule. This technique can be shown to be equivalent to using a piecewise-linear finite-element discretization. Using edge-based data structures, the path integrals can be cast as a loop over the edges, even in three dimensions as shown by Mavriplis [2]. For dissipative terms, a blend of Laplacian and biharmonic operators is employed, the Laplacian term acting only 
in the vicinity of shocks. A multi-stage Runge-Kutta scheme is used to advance the solution in time. In addition, local time stepping, enthalpy damping and residual averaging are used to accelerate convergence. In previous work $[1,2]$, as well as in the present work, only the Laplacian dissipative term (with constant coefficient) is used on the coarse grids. Thus, the fine grid solution itself is second order accurate, while the solver is only first order accurate on the coarse grids.

\section{Details of agglomeration}

The object of agglomeration is to derive coarse grids from a given fine grid. In graph theoretical terms, if the initial grid is interpreted as a graph, the agglomeration problem is that of finding a maximal independent set with certain desirable properties. A subset of the vertices of a graph is termed an independent set if no two vertices in the set are adjacent. An independent set is maximal if any vertex not in the set is dominated by(adjacent to) at least one vertex in it. A desirable property for the coarse grids in multigrid is that the number of grid cells should decrease by a nearly constant factor when moving from a fine to a coarse grid. This factor is 4 in two dimensions and 8 in three dimensions. The graph problem reduces to finding the maximal independent graph with the minimum cardinality(size) and is $\mathrm{nP}$ complete. Hence, a heuristic algorithm is used to derive the coarse grids.

The agglomeration is accomplished by using a greedy-type frontal algorithm. It is a variation on the one used by Lallemand et al. [8]. We call this the isotropic version, since it fuses in Step 2 all the neighboring fine grid control volumes that are not already fused. It is outlined below:

1. Pick a starting vertex on one of the surface elements.

2. Agglomerate the control volumes associated with its neighboring vertices which are not already agglomerated.

3. Define a front as comprised of the exterior faces of the agglomerated control volumes. Place the exposed edges (duals to the faces) in a queue.

4. Pick the new starting vertex as the unprocessed vertex incident to a new starting edge which is chosen from the following choices given by order of priority:

- An edge on the front that is on the solid wall.

- An edge on the solid wall.

- An edge on the front that is on the far field boundary.

- An edge on the far field boundary.

- The first edge in the queue.

5. Go to Step 2 until the control volumes for all vertices have been agglomerated.

There are many other ways of choosing the starting vertex in Step 4 of the algorithm, but we have found the above strategy to be the best. The efficacy of the agglomeration 
algorithm can be characterized by a histogram of the number of fine grid cells comprising each coarse grid cell. In two dimensions, ideally, each coarse grid cell will be made up of exactly four fine grid cells. The various strategies can be evaluated by how close they come to this ideal situation. One variation is to pick the starting edge randomly from the edges currently on the front. Figure 1 shows a plot of the number of coarse grid cells as a function of the number of fine grid cells they contain for a triangular mesh with 11340 vertices, with the agglomeration algorithm described above, and with the variant. It is clear that our agglomeration algorithm is superior to the variant. The number of singletons (coarse grid cells having exactly one fine cell) is also much smaller with our algorithm compared to the variant. We have also investigated another variation of the algorithm where the starting vertex in Step 4 is randomly picked from the field; this turns out be much worse. It is possible to identify the singleton cells and agglomerate them with the neighboring cells, but this has not been done. Finally, it should be noted that with the isotropic version of the algorithm, one has no control over the shapes or the aspect ratios of the coarse grid cells.

In order to have some control over the aspect ratios of the coarse grid cells, we have developed a variant loosely termed the semi-coarsened version. This is similar in spirit to semi-coarsening for highly stretched structured grids, where the coarsening is done in the direction of stretching. The algorithm produces more uniform coarse grids and is expected to be beneficial especially for viscous calculations. This algorithm is a combination of breadthfirst and depth-first searches and is more expensive than the isotropic version. In the semicoarsened version, Steps 2 and 3 of the isotropic algorithm are replaced by the following steps:

1. For each starting vertex, form a list of neighboring control volumes.

2. Fuse the control volume from the list that maximizes the aspect ratio of the coarse grid cell defined as $V / S^{n}$, where $\mathrm{V}$ is the volume, $\mathrm{S}$ is the surface area and $n$ is 2 in two dimensions and 1.5 in three dimensions.

3. Update the front by adding the exposed edges (duals to the exposed faces) to the queue.

4. Update the list of neighboring control volumes.

5. Go to Step 2 until a prespecified number of control volumes are fused into a coarse grid cell.

We compare the two versions of the agglomeration algorithm by inspecting the coarse grids obtained on a small test problem. The fine grid about an NACA0012 airfoil contains 4224 vertices. The third-level coarse grids obtained with the isotropic and the semi-coarsened algorithms are displayed in Figures 2 and 3, and they contain 80 and 72 cells, respectively. The grid obtained with the semi-coarsened version is more uniform and has more favorable aspect ratios.

A three-dimensional application of the isotropic agglomeration algorithm is shown in Figures $4(\mathrm{a}-\mathrm{c})$. The fine grid about an ONERA M6 wing contains 9428 vertices. Figure $4 \mathrm{a}$ shows the dual to the surface grid. The coarse grids contain 1466, 253 and 46 vertices, respectively. Figures $4 \mathrm{~b}$ and $4 \mathrm{c}$ show the level- 1 and level- 3 surface grids. 
The procedures outlined above are applied recursively to create coarser grids. The boundaries between the control volumes on the coarse grids are composed of the edges/faces of the fine grid control volumes. In two dimensions, we have observed that the number of such edges only decreases by a factor of 2 when going from a fine to a coarse grid, even though the number of vertices decreases by a factor of 4 . Since the computational load is proportional to the number of edges, this is unacceptable in the context of multigrid. However, if we recognize that the multiple edges separating two control volumes can be replaced by a single edge connecting the end points, then the number of edges does go down by a nearly a factor of 4. This is illustrated in Figure 5 which shows the fine grid edges (solid lines), fine grid dual edges (dashed lines) and the edges for the coarse grid control volumes (thick solid lines). The edges connecting A and B can be effectively replaced by a single straight edge shown by the thick dashed line. A similar approximation can be made in three dimensions as well. Since only a first order discretization is used on the coarse grids, there is no approximation involved in this step. If a flux function that involved the geometry in a nonlinear fashion were used, such as Roe's approximate Riemann solver [11], this is still a very good approximation. As a result of this approximation, the work decreases by nearly a constant factor when moving from a fine to a coarse grid.

Both versions of the algorithm have been optimized to run in linear time. They employ hashing to combine the multiple fine grid control volume faces separating two coarse grid cells into one planar face. This has enabled us to run very large three-dimensional problems (tetrahedral grids with over 800,000 vertices) in a reasonable amount of time. The semicoarsened version is more expensive than the isotropic version, but creates more uniform grids. The three-dimensional versions of the algorithm require 1200 bytes of memory per fine grid vertex on a Sun Sparc 10 workstation. The times required to create four coarse grids by agglomeration from a three-dimensional tetrahedral grid with 50,000 fine grid vertices on this workstation are 63 and 128 seconds with the isotropic and the semi-coarsened versions, respectively. The output of the agglomeration is a list of fine grid cells belonging to each coarse grid cell and edge coefficients, which represent the projected areas of the planar contol-volume faces onto the coordinate planes.

\section{Details of multigrid}

The base scheme needs to be modified to handle arbitrary polyhedral coarse grids. The time step calculation and the computations of the Euler and dissipative fluxes are recast in such a way as to only utilize the volumes and the edge coefficients. In the original scheme, an isotropic dissipative coefficient based on the spectral radius integrated over the control volume faces was used. For very coarse and highly nonuniform polyhedral grids, we have had to use a dissipative coefficient based on the spectral radius for each face in order to obtain stable coarse grid operators. Another way to ensure a stable coarse grid operator is to formulate a first order Roe's upwind solver for polyhedral grids [12], but was found to be considerably more expensive [13].

Since the fine grid control volumes comprising a coarse grid control volume are known, the restriction is similar to that used for structured grids. The residuals are simply summed from the fine grid cells and the variables are interpolated in a volume-weighted manner. For 
the prolongation operator, we use a simple injection (a piecewise-constant interpolation). This is an unfortunate but an unavoidable consequence of using the agglomeration strategy. A piecewise-linear prolongation operator implies a triangulation, the avoidance of which is the main motivation for the agglomeration. In actuality, the agglomeration procedure itself implies a triangulation. In inspecting Figure 5 which shows an agglomerated coarse grid, we notice that three dual edges meet at every vertex, such as A or B. Thus, each vertex in this figure represents a triangle made up of the three vertices representing the three coarse grid cells. However, we have found this triangulation to be invalid in general with a number of crossing grid lines, especially near the trailing edges of the airfoils. Another possible way to achieve a piecewise-linear interpolation is to utilize the gradient that is computed in a least squares sense by making use of data from some defined neighborhood of the coarse grid cell. We do not follow either of these approaches because of their associated complexity. The adverse impact of the injection is minimized by employing additional smoothing steps. This is achieved by applying an averaging procedure to the injected corrections. In an explicit scheme, solution updates are directly proportional to the computed residuals. Thus, by analogy, for the multigrid scheme, corrections may be smoothed by a procedure previously developed for implicit residual smoothing [1]. The implicit equations for the smoothed corrections are solved using two iterations of a Jacobi scheme after prolongation at each grid level.

The agglomeration procedure can be interpreted as a Galerkin coarse grid approximation [14]. If the fine grid operator can be written as

$$
A u=f,
$$

the Galerkin coarse grid approximation can be derived as

$$
\bar{A} \bar{u}=\bar{f}
$$

with

$$
\bar{A}=R A P,
$$

and

$$
\bar{f}=R f .
$$

Here $R$ and $P$ are the restriction and prolongation operators, $u$ is the fine grid vector and $\bar{u}$ is the coarse grid vector. In the case of agglomeration, both $R$ and $P$ are chosen to be piecewise-constant operators. Note that the scaling of $R$ is immaterial in this formulation. Agglomeration can thus be viewed as identifying all the fine grid cells with the coarse grid cell to which they belong, and then summing the equations corresponding to these cells. Interpreted in this manner, agglomeration multigrid is similar to algebraic multigrid [15] except that the equations to be combined are prespecified.

\section{Results and discussion}

Representative two- and three-dimensional flows are computed over geometries with varying degrees of geometrical complexity. First, results are presented for a two-dimensional flow 
problem. The performance of the agglomerated multigrid algorithm is compared with that of the non-nested multigrid algorithm [1,2]. Inviscid flow over a four-element airfoil in landing configuration is computed. The freestream Mach number is 0.2 and the angle of attack is $5^{\circ}$. The fine grid has 11340 vertices and its dual is shown in Figure 6. The coarse grids for use with the non-nested multigrid algorithm (not shown) contain 2942 and 727 vertices. It was not possible to generate coarser grids for this complex geometry. Four agglomerated coarse grids obtained using the isotropic version of the algorithm are shown in Figure 7. These grids contain 3027, 822, 217 and 63 vertices (regions), respectively. The convergence histories of the non-nested and agglomeration multigrid algorithm are shown in Figure 8. The convergence histories of the three-level multigrid are comparable but the convergence is slightly better with the agglomerated multigrid strategy. This is a bit surprising since the original multigrid algorithm employs a piecewise-linear prolongation operator. A possible explanation is that the coarse grids created by the agglomeration algorithm are better than those employed in the non-nested algorithm. Convergence improves considerably with the 5-level multigrid algorithm. The CPU times required on the Cray Y-MP for the 3-level multigrid are are 59 and 60 seconds with the original and the agglomerated algorithms, respectively. The CPU time required for the 5 level agglomeration multigrid is 73 seconds. The convergence rate per work unit with the 3 -level non-nested multigrid algorithm is 0.940 . The convergence rates per work unit with the 3 -level and 5-level agglomerated multigrid algorithm are 0.938 and 0.920 , respectively. We also observe in Figure 8 that there is only a slight difference in convergence rates between the two versions of the agglomeration algorithm. The convergence rates per multigrid cycle with the isotropic and semi-coarsened versions of the agglomeration algorithm are 0.898 and 0.894 , respectively for the 5 -level multigrid scheme. Similar behavior has been observed in most of our two- and three-dimensional computations. This is not surprising since the aspect ratios of the cells for these Euler grids are quite benign. The payoff, we believe, will be in the computation of viscous flows, where cell aspect ratios of 1000 are typical. Nevertheless, in the rest of the section, all the coarse grids have been derived using the semi-coarsened version of the algorithm.

We next present results from three-dimensional calculations of flows over geometries of increasing complexity. The first flow considered is transonic flow over an ONERA M6 wing at a freestream Mach number of 0.84 and an angle of attack of $3.06^{\circ}$. The geometry consists of the wing delimited by a symmetry plane. The mesh was generated using the advancingfront grid generation technique [16]. The grid contains 357,900 vertices and just over 2 million tetrahedra. However, due to limitations in printing resolution, we only display in Figure 9 the surface mesh corresponding to a coarser grid with 53,961 vertices. The semi-coarsened agglomeration algorithm is used to generate five coarse grids. These coarse grids contain $62594,13033,3105,804$, and 54 vertices, respectively. Figure 10 shows the Mach contours on the surface where the familiar double shock pattern for this standard test case may be observed. The convergence rate of 0.878 per cycle is achieved which is comparable to the rate of 0.871 achieved by Mavriplis [2] with 4-level non-nested full multigrid procedure for the same case. The final solution is identical to that obtained by Mavriplis [2] since the same fine mesh discretization is employed. This particular solution has also been shown to be in good agreement with other inviscid computations for this case [2]. The convergence history for the 6-level multigrid using the five agglomerated coarse grids is shown in Figure 11. The residuals are reduced by six orders of magnitude in $100 \mathrm{~W}$-cycles, which required 
55 minutes of CPU time and $44 \mathrm{MW}$ of memory on the Cray Y-MP/1. By comparison, the single grid run resulted in a residual reduction of merely 3 orders of magnitude in 400 cycles. A complete multigrid cycle requires about $80 \%$ more CPU time than the driving single grid cycle and a $30 \%$ increase in memory requirements.

The next case involves the solution of inviscid transonic flow over a low-wing transport configuration. The geometry consists of a half fuselage bounded b: a s; netry plane with a wing and a nacelle. The fine mesh for this case contains 804,056 vertices and approximately 4.5 million tetrahedra. Again, due to limitations in prining resolution, only the surface mesh for a coarser mesh with 106,064 vertices is depicted in Figure 12. This computation represents one of the largest unstructured grid cases attempted to date. However, this is believed to be representative of the type of resolution required for engineering calculations for configurations of this type. Six coarse grids are derived by the semi-coarsened agglomeration algorithm and contain respectively, 132865, 25489, 5678, 1421, 373 and 99 vertices. The freestream conditions are Mach number of 0.77 and and $1.116^{\circ}$ incidence. The convergence history using a seven-level agglomerated multigrid strategy is shown in Figure 13. The residuals are reduced by over 6 orders of magnitude in $100 \mathrm{~W}$-cycles, which corresponds to a convergence rate of 0.876 per cycle. This compares favorably with the rate of 0.870 obtained using a 5-level non-nested multigrid strategy for the same case. The agglomerated multigrid computation required $96 \mathrm{MW}$ of memory and 90 minutes of CPU time on a Cray Y-MP/1 computer. The computed Mach contours are depicted in Figure 14 where the upper surface wing shock pattern is evident.

Finally, we consider flow over a fuselage-wing-flap geometry. This case, which has the additional complexity of a flap system, is representative of a simplified Boeing 737 landing configuration geometry. The fine grid contains 134,539 vertices and the surface mesh is shown in Figure 15. This mesh was provided by the author of reference [17]. While the grid is too coarse to provide accurate engineering results for this geometry, this case has been included to illustrate the power of the agglomeration technique in handling complex geometries. The small gap sizes between neighboring flap elements restrict the effectiveness of traditional multigrid methods. In the non-nested mesh approach for example, the coarsest meshes must contains cells small enough to resolve these gaps, or risk altering the geometry. For the agglomeration approach, on the other hand, these restrictions are completely removed. We have constructed five coarse agglomerated grids of 22238,4043, 810, 205 and 52 vertices. The topology of the geometry is allowed to change on these coarse grids; the interpretation of agglomeration as an algebraic technique (cf. Equation (3)) guarantees the validity of the procedure in this case. For a freestream Mach number of 0.4 and $0^{\circ}$ incidence, the 6-level agglomeration strategy results in a residual reduction of 5.5 orders of magnitude in 100 W-cycles for a convergence rate of 0.885 per cycle. This rate is consistent with the rates obtained in the previous cases. The convergence history for this case is displayed in Figure 16. It should be noted that engineering accuracy for the cases shown in this paper can be achieved in fewer than 50 multigrid cycles. 


\section{Conclusions}

It has been shown that the agglomeration multigrid strategy can be made to approximate the efficiency of the unstructured multigrid algorithm using independent, non-nested coarse meshes, both in terms of convergence rates and CPU times. It is further shown that arbitrarily coarse grids can be obtained with the agglomeration technique, although care must be taken to ensure that the coarse grid operator is convergent on these grids. The agglomeration itself is done very efficiently as a preprocessing step. It has been shown that the agglomeration algorithm can be tailored to achieve desired coarse grid properties. Applications to flows over complex configurations have been provided to illustrate the power and flexibility of the agglomeration multigrid strategy.

\section{References}

[1] Mavriplis, D. J., and Jameson, A., "Multigrid Solution of the Two-Dimensional Euler Equations on Unstructured Triangular Meshes", AIAA Journal, Vol 26, No. 7, July 1988, pp. 824-831.

[2] Mavriplis, D. J., "Three Dimensional Multigrid for the Euler equations", Proc AIAA 10th Comp. Fluid Dynamics Conf., Honolulu, Hawaii, June 1991, pp. 239-248.

[3] Leclercq, M. P., "Resolution des Equations d'Euler par des Methodes Multigrilles Conditions aux Limites en Regime Hypersonique", Ph.D Thesis, Applied Math, Universite de Saint-Etienne, April 1990.

[4] Peraire, J., Peiro, J., and Morgan, K., "A 3D Finite-Element Multigrid Solver for the Euler Equations", AIAA paper 92-0449, January 1992.

[5] Perez, E., "A 3D Finite-Element Multigrid Solver for the Euler Equations", INRIA Report No. 442, September 1985.

[6] Connell, S. D., and Holmes, D. G., "A 3D Unstructured Adaptive Multigrid Scheme for the Euler Equations", AIAA Paper 93-3339-CP, July 1993.

[7] Guillard, H., "Node Nested Multigrid with Delaunay Coarsening”, INRIA Report No. $1898,1993$.

[8] Lallemand, M., Steve, H., and Dervieux, A., "Unstructured Multigridding by Volume Agglomeration: Current Status", Computers and Fluids, Vol. 21, No. 3, pp. 397-433, 1992.

[9] Smith, W. A., "Multigrid Solution of Transonic Flow on Unstructured Grids", Recent Advances and Applications in Computational Fluid Dynamics, Proceedings of the ASME Winter Annual Meeting, Ed. O. Baysal, November 1990.

[10] Spragle, G., Smith, W. A., and Yadlin, Y., "Application of an Unstructured Flow Solver to Planes, Trains and Automobiles", AIAA Paper 93-0889, January 1993. 
[11] Roe, P. L., "Characteristic-based Schemes for the Euler Equations", Annual Review of Fluid Mechanics, Vol. 18, 1986, pp. 337-365.

[12] Barth, T.J., and D. Jespersen, "The design and application of upwind schemes on unstructured meshes", AIAA Paper 89-0366, January 1989.

[13] Venkatakrishnan, V., Mavriplis, D.J., and Berger,M.J., "Unstructured Multigrid through Agglomeration", to appear in the Proceedings of the 6th Copper Mountain Multigrid Conference, April 1993.

[14] Wesseling, P., "An Introduction to Multigrid Methods", John Wiley \& Sons, 1992.

[15] Multigrid Methods, McCormick, S. ed, SIAM Frontiers in Applied Mathematics, SIAM, Philadelphia, 1987.

[16] Parikh, P., Pirzadeh, S., and Lohner, R., "A Package for 3-D Unstructured Grid Generation, Finite-element Solution and Flow-visualization", NASA CR-182090, September 1990.

[17] Dodbele, S.S., "Three-dimensional Aerodynamic Analysis of a High-lift Transport Configuration", AIAA Paper 93-3536, AIAA Appiled Aerodynamic Conference, Monterey, CA, August 1993. 


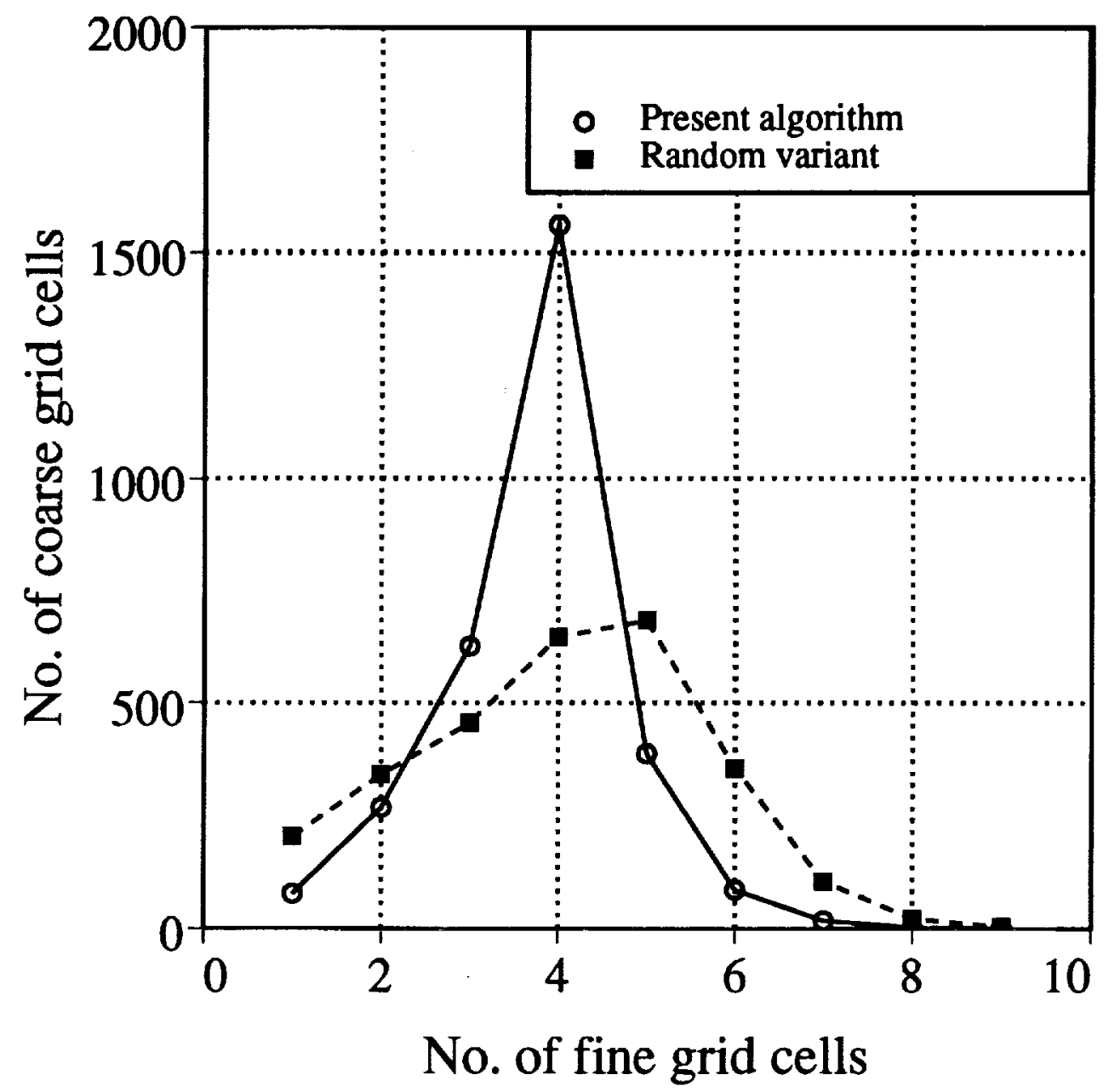

Figure 1: Number of coarse grid cells vs the number of fine grid cells they contain. 


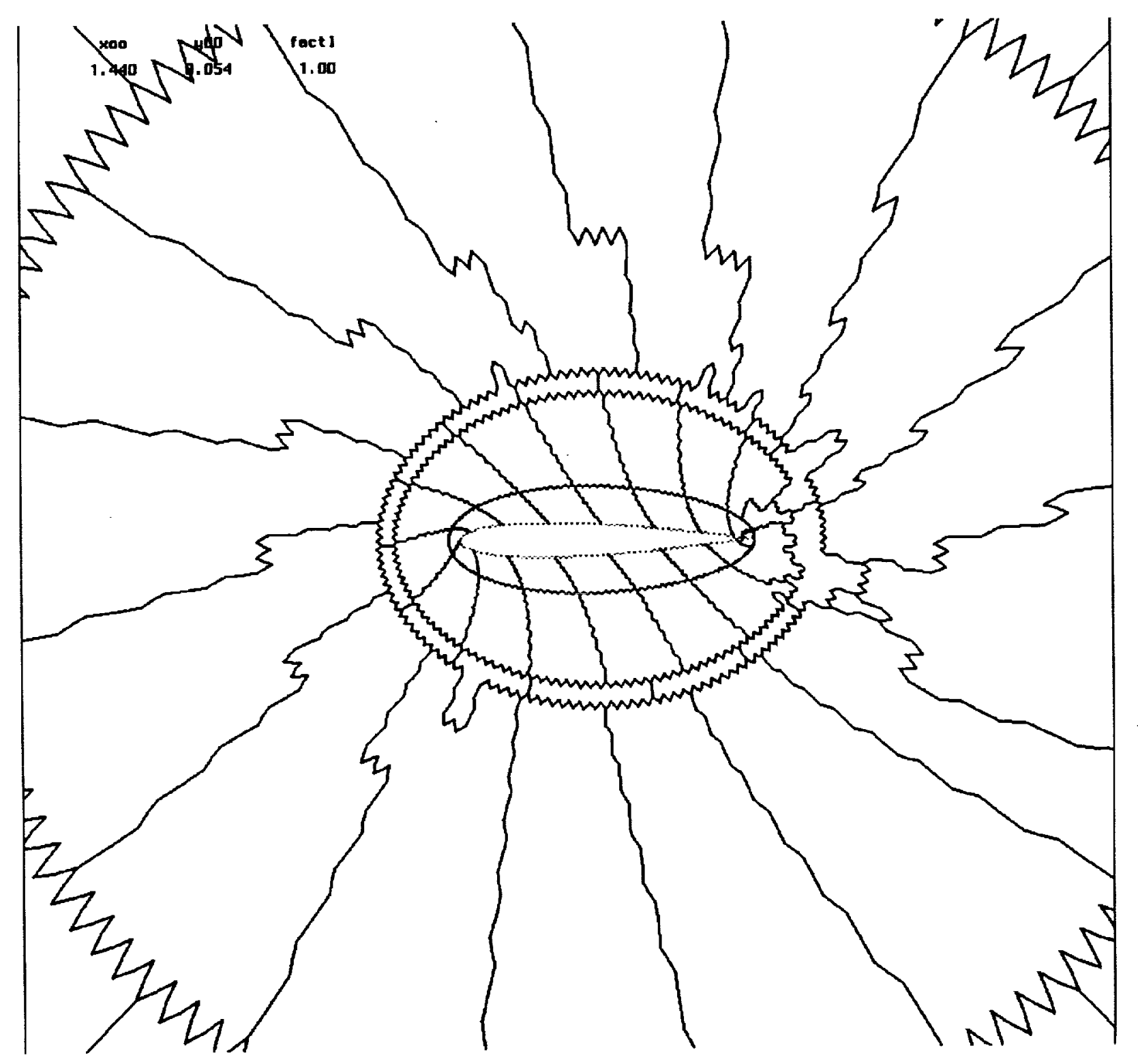

Figure 2: Third-level coarse grid obtained with the isotropic agglomeration algorithm for a fine grid with 4224 vertices. 


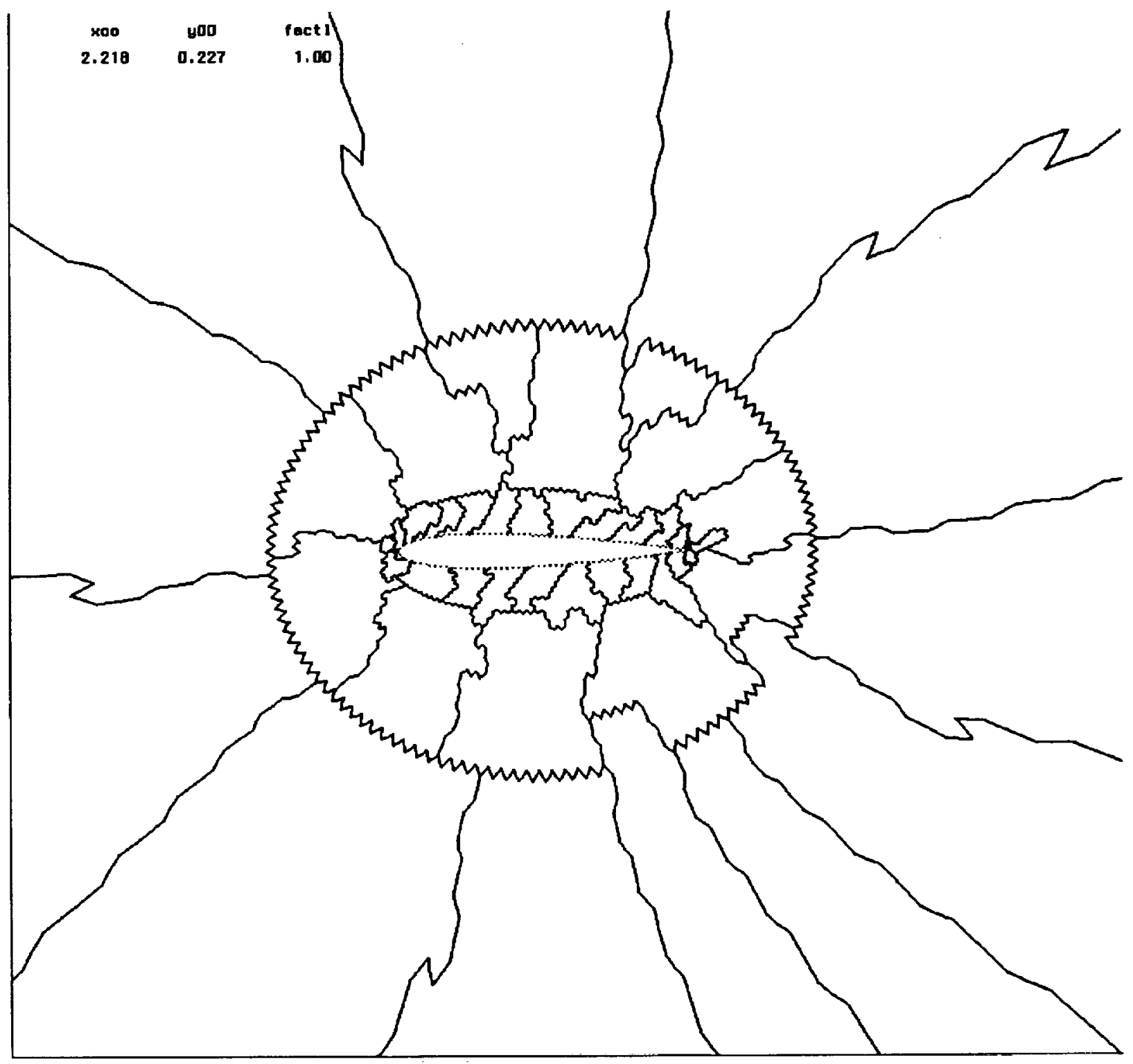

Figure 3: Third-level coarse grid obtained with the semi-coarsened agglomeration algorithm for a fine grid with 4224 vertices. 

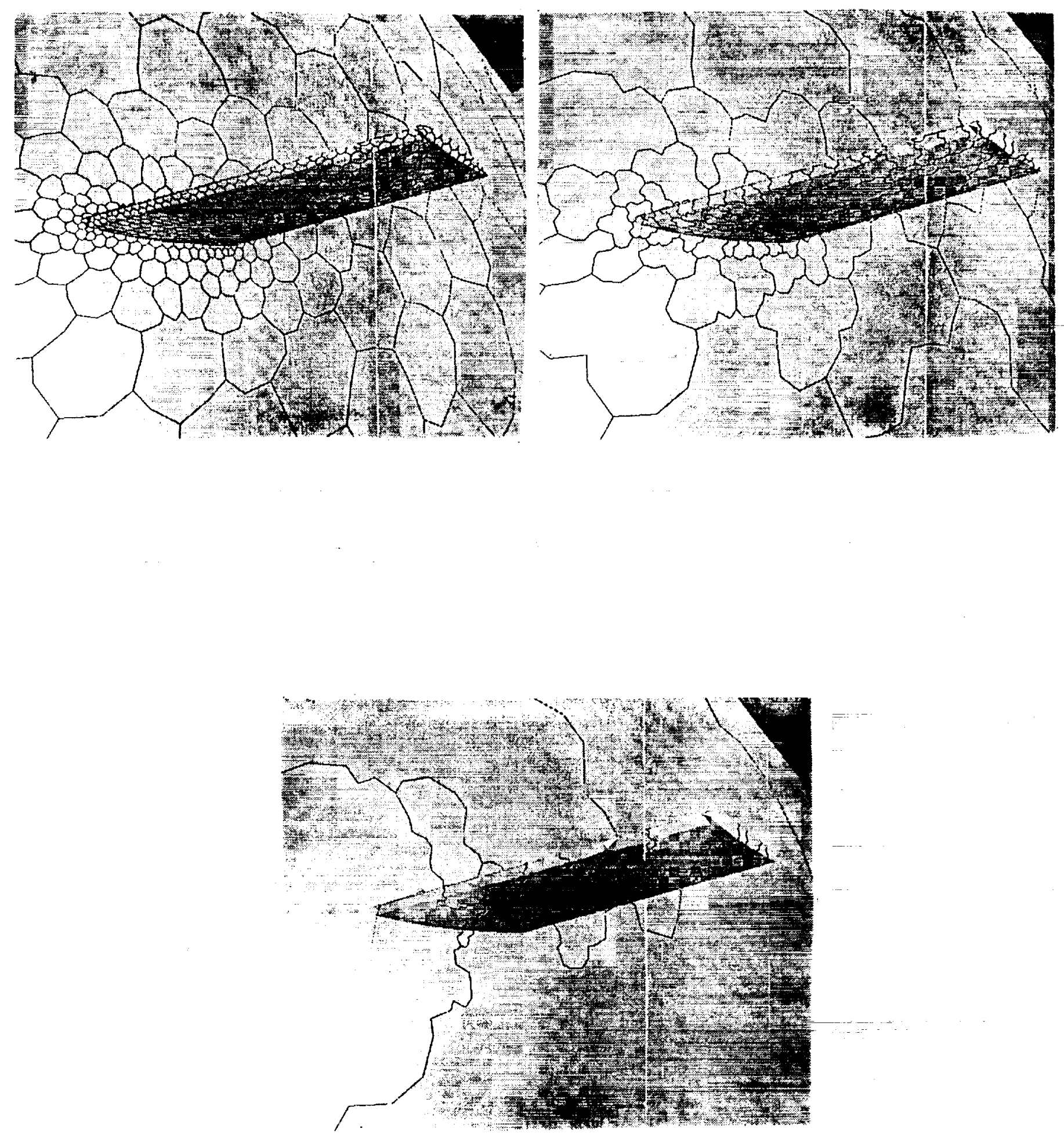

Figure 4: Surface grids (a) Dual to the fine grid having 9428 vertices (b) Level-1 coarse grid (c) Level-3 coarse grid 


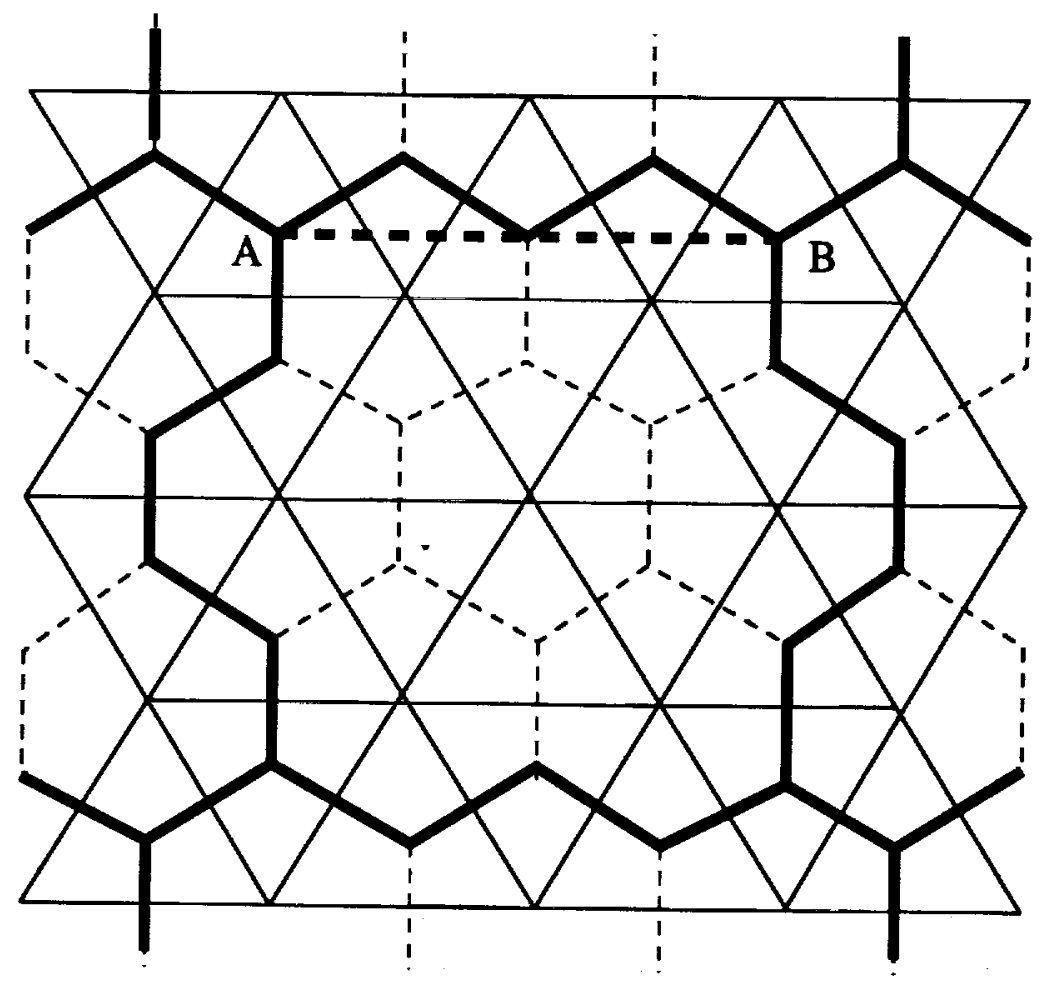

Figure 5: An example of an agglomerated coarse grid. 


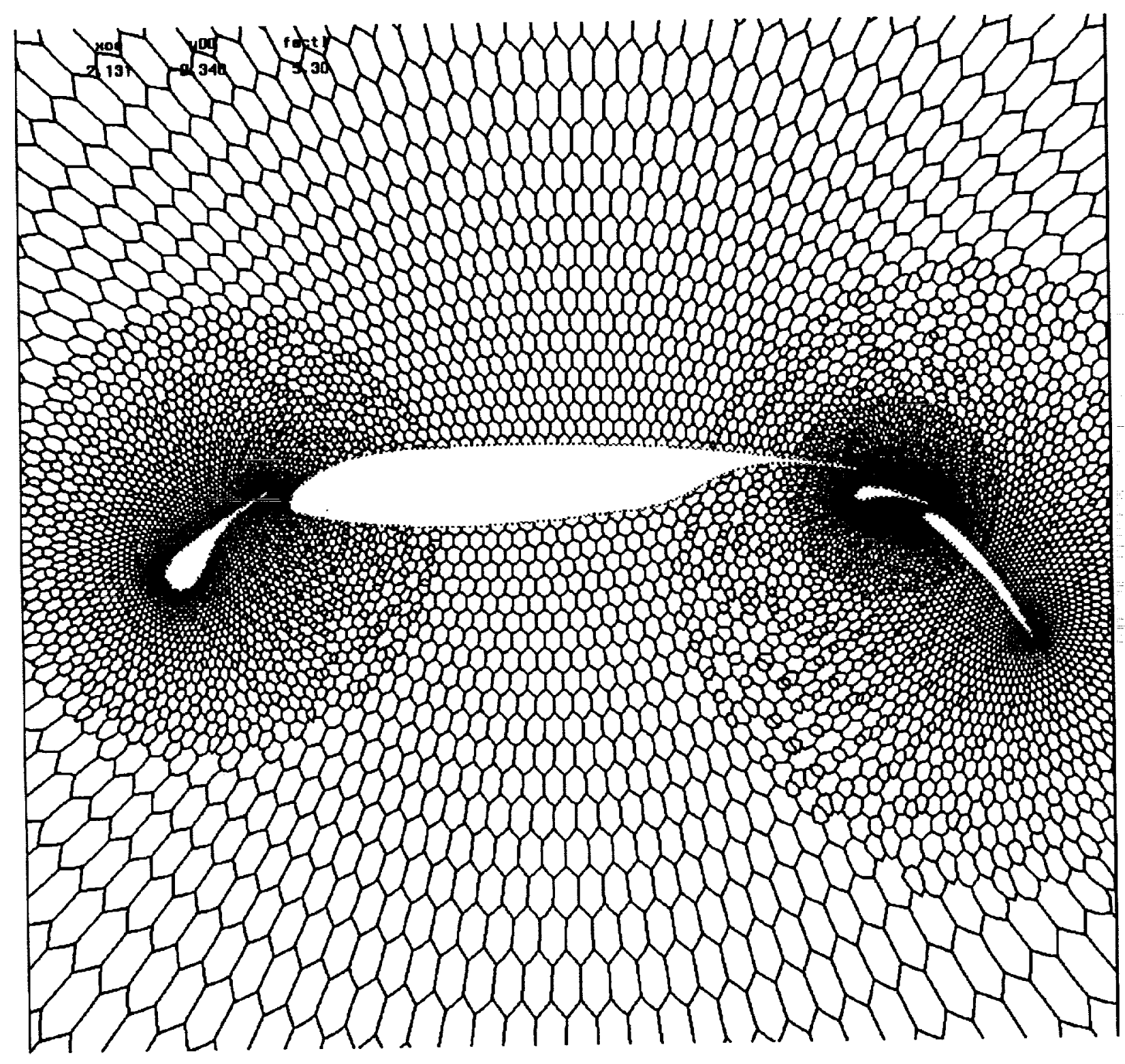

Figure 6: Dual to the fine grid having 11340 vertices about a 4-element airfoil. 

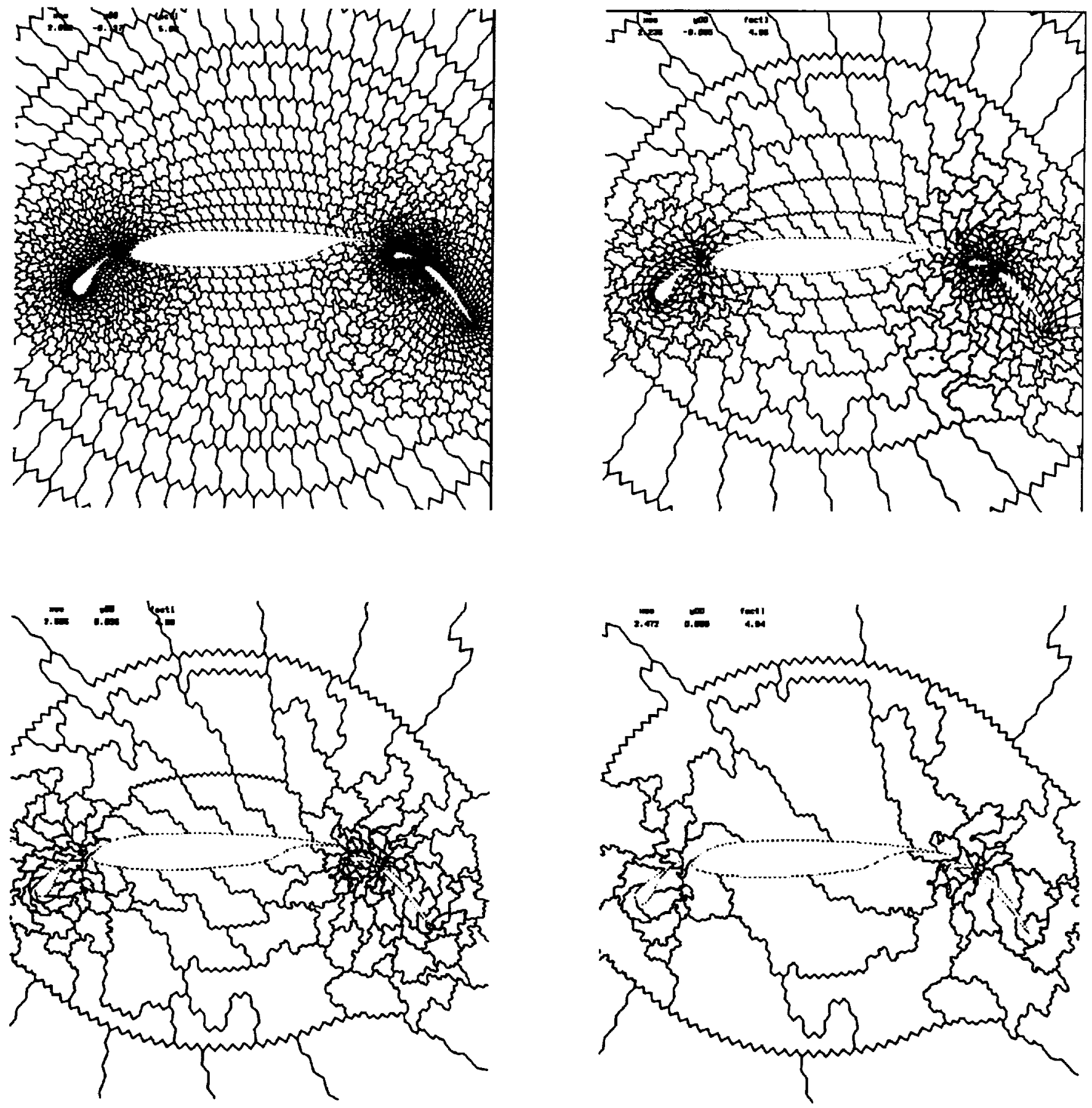

Figure 7: Four agglomerated coarse grids for the four-element test case. 


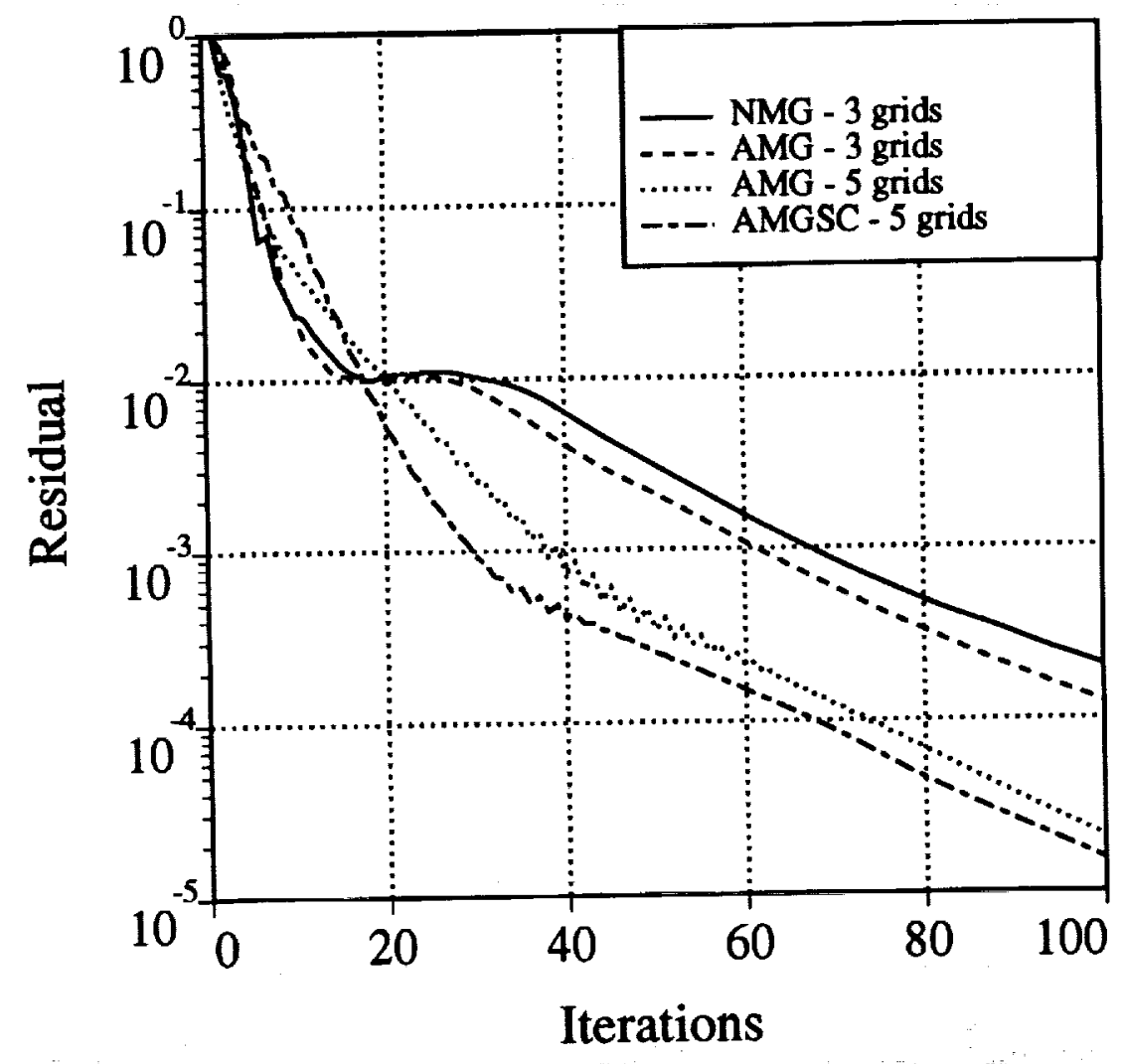

Figure 8: Convergence histories with the agglomerated and original multigrid. NMG - Nonnested multigrid; AMG - Agglomerated multigrid using the isotropic algorithm; AMGSC Agglomerated multigrid using the semi-coarsened algorithm. 


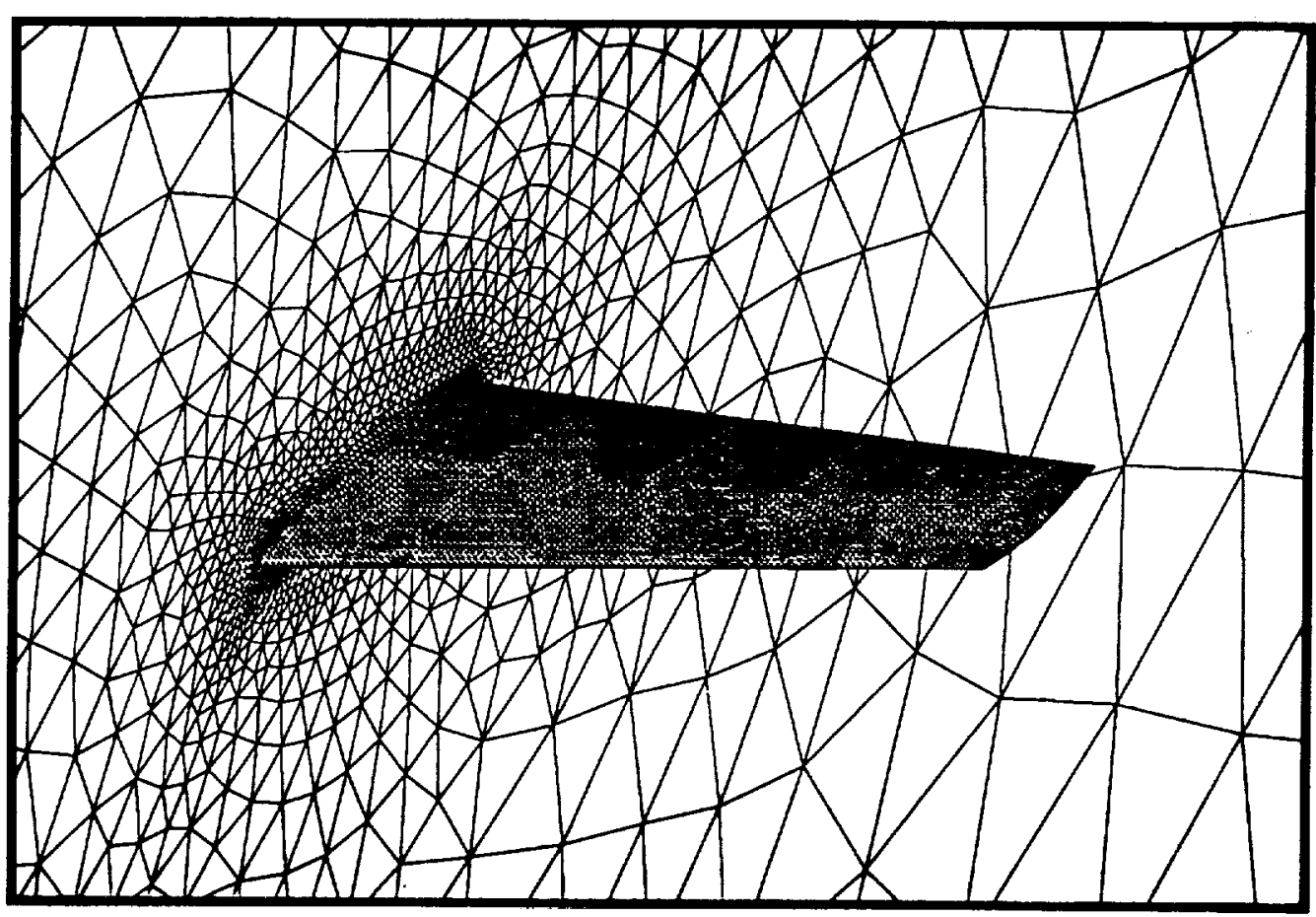

Figure 9: Surface mesh for a coarse grid with 53,961 vertices about an ONERA M6 wing. The fine mesh has 357,900 vertices. 


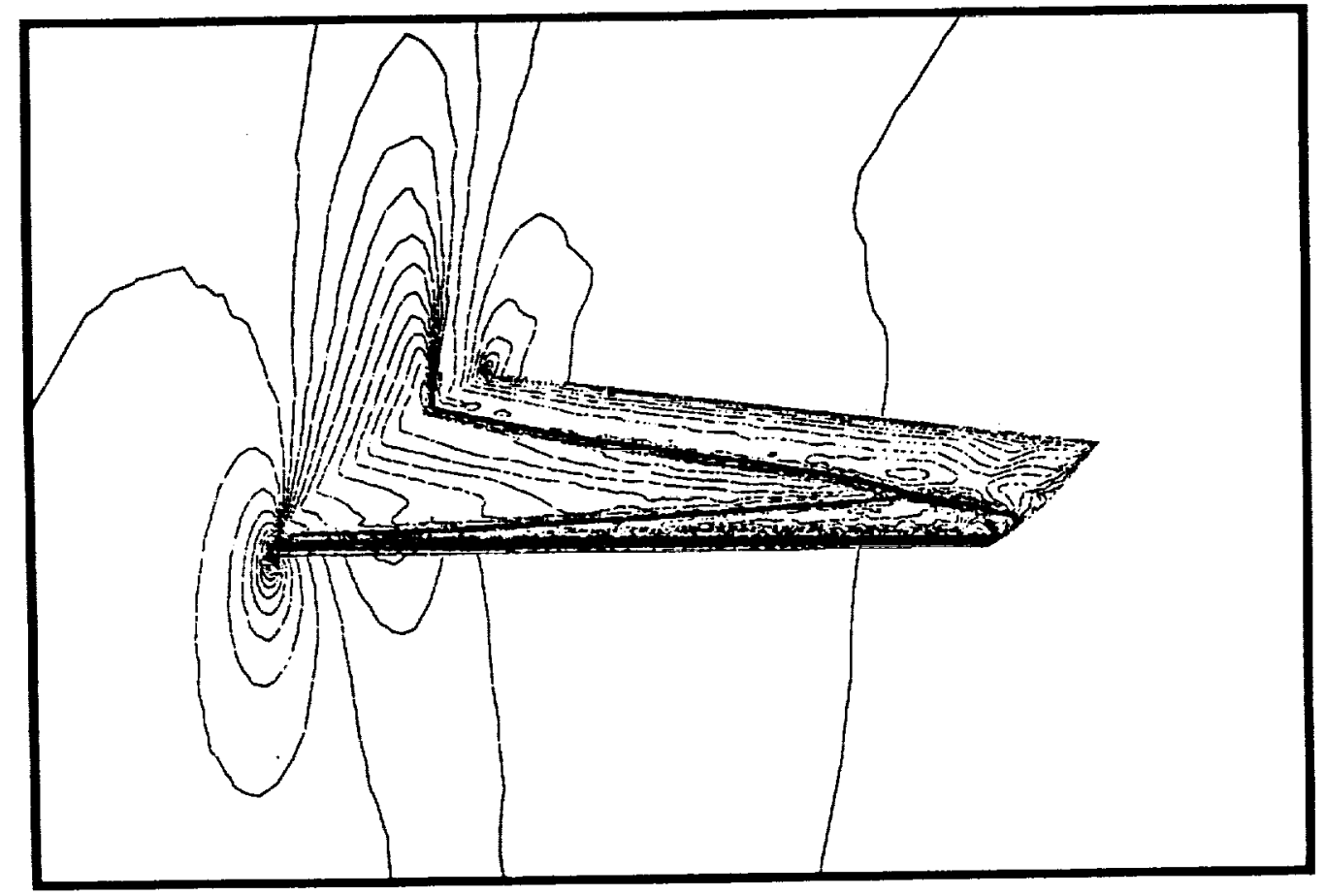

Figure 10: Mach contours on the surface for transonic flow over the ONERA M6 wing using the fine grid. 


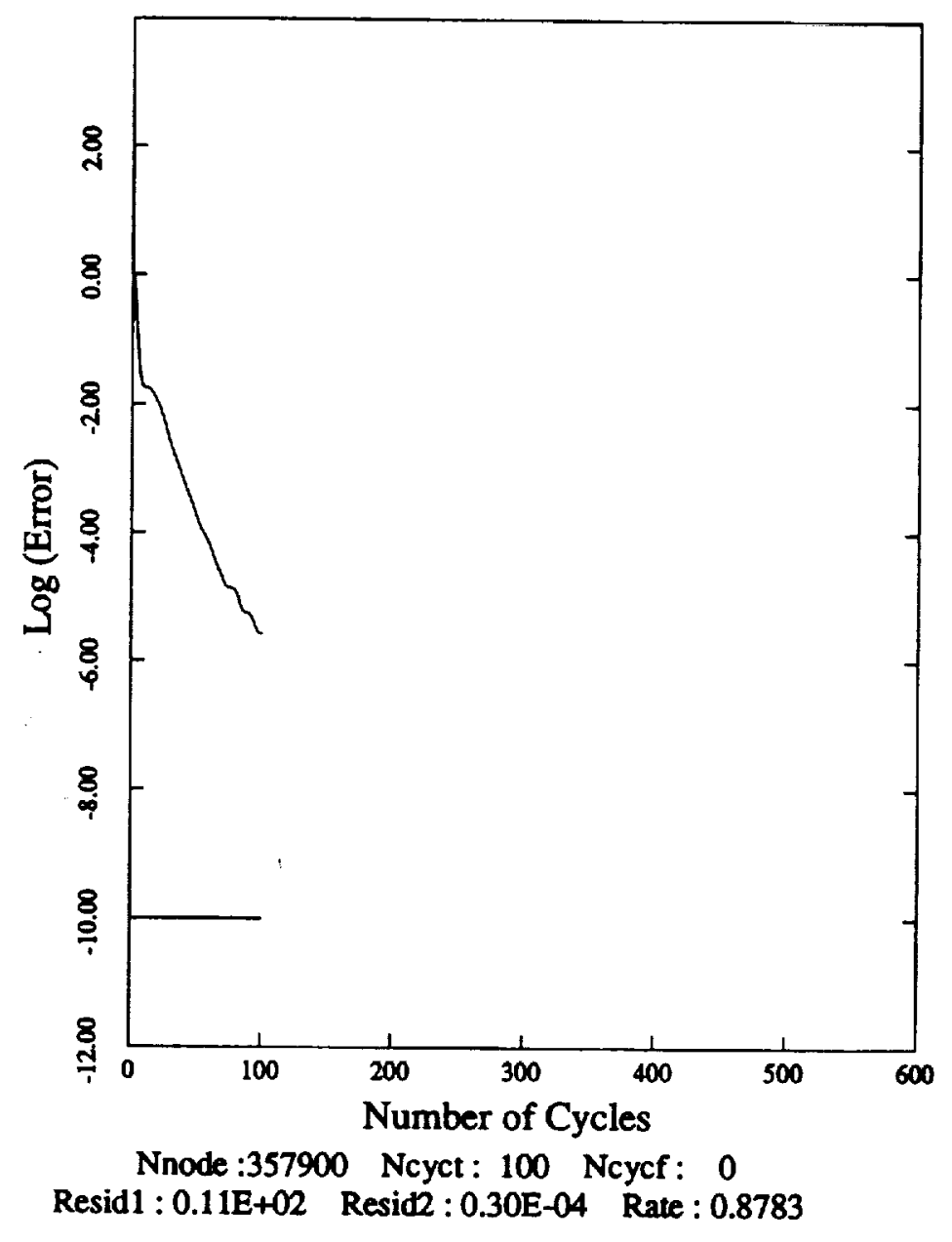

Figure 11: Convergence history of the 6-level agglomerated multigrid scheme for the ONERA M6 wing case. 


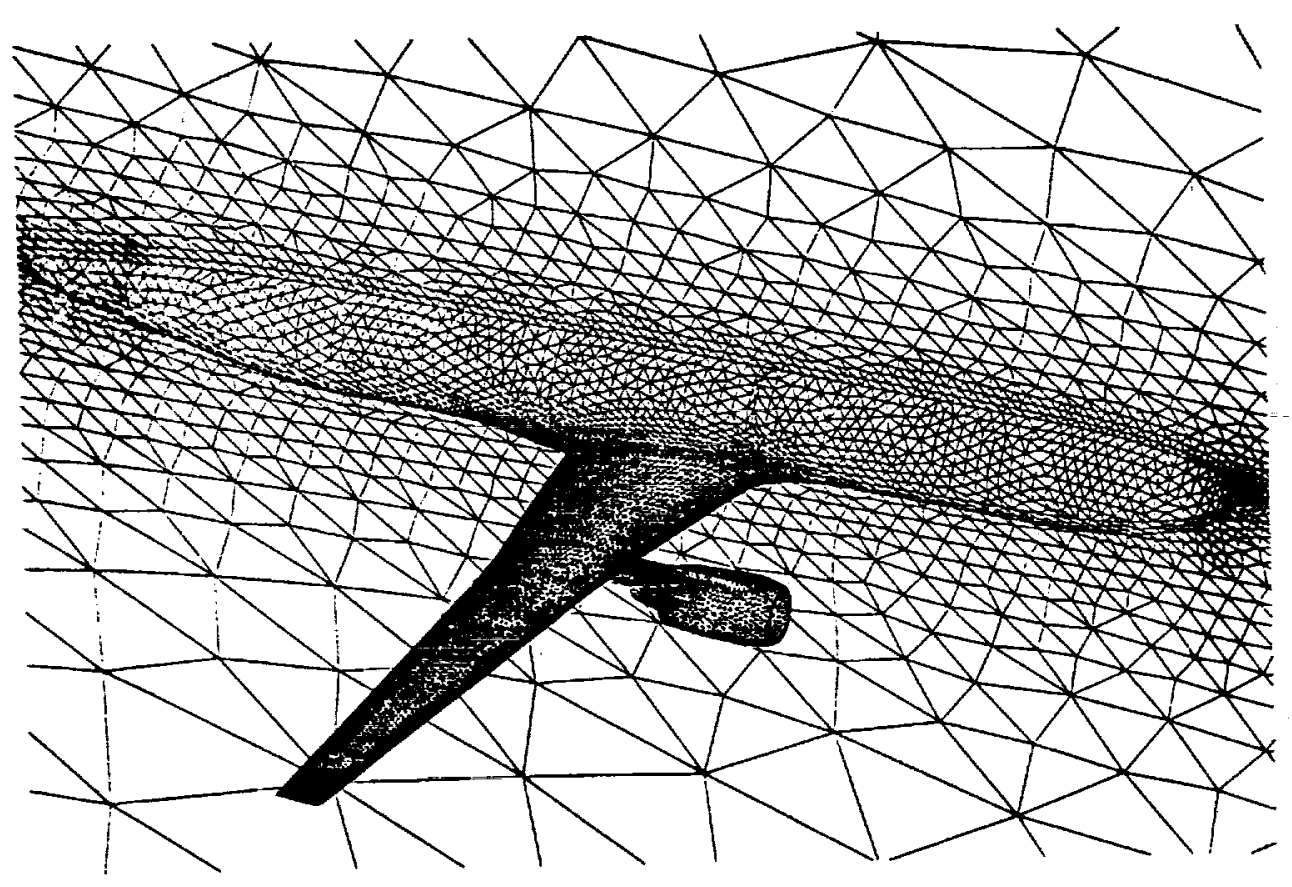

Figure 12: Surface mesh for a coarse grid with 106,064 vertices about a low-wing transport configuration. The fine mesh has 804,056 vertices. 


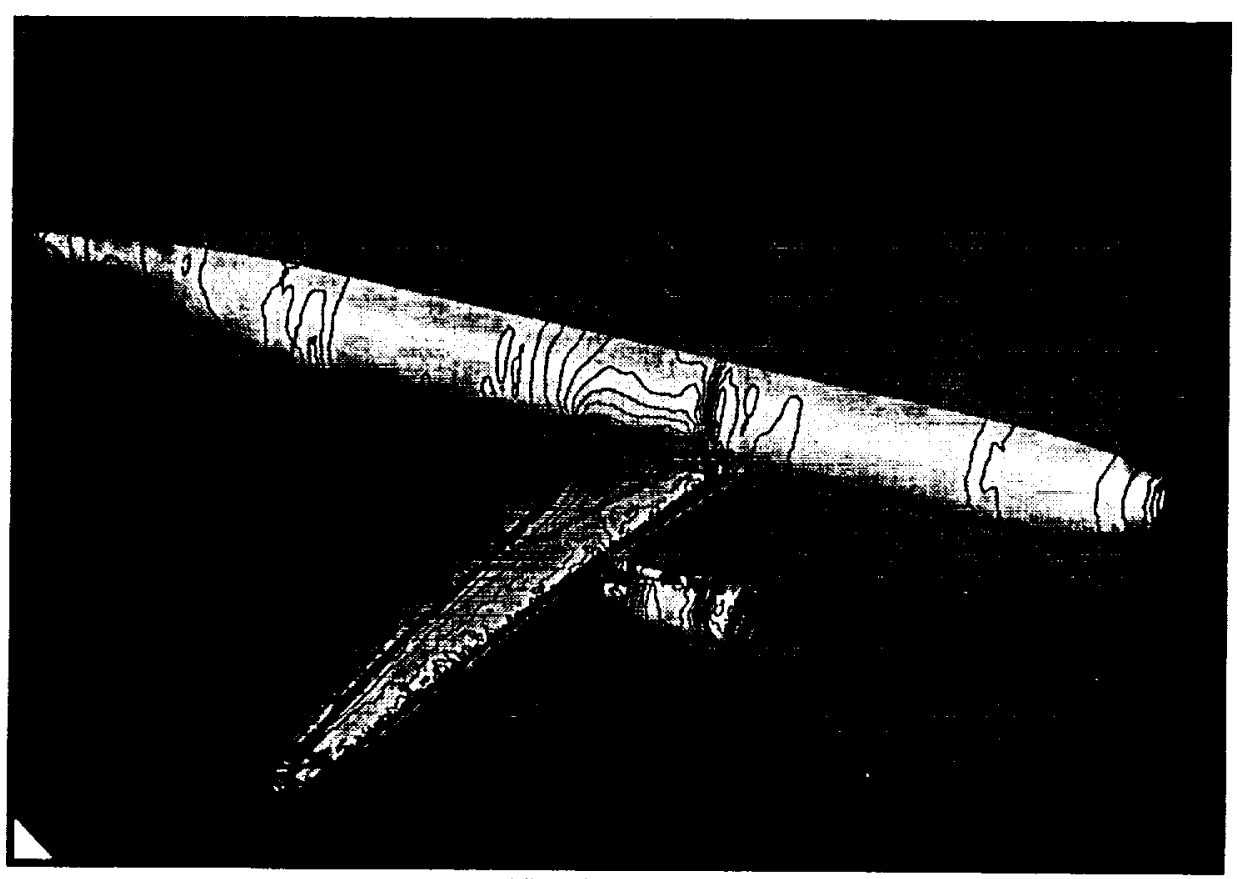

Figure 13: Mach contours on the surface for transonic flow over the low-wing transport configuration. 


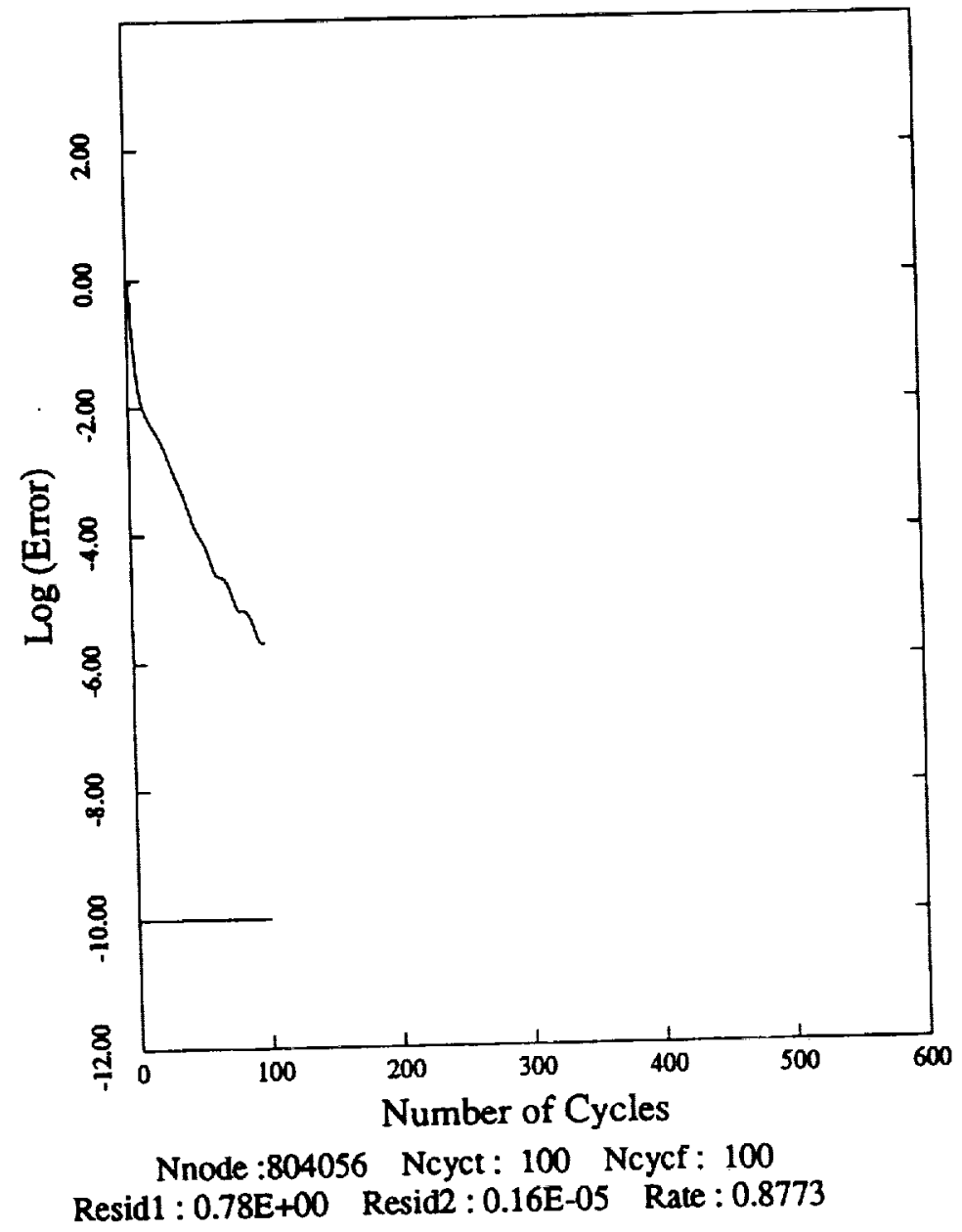

Figure 14: Convergence history of the 7-level agglomerated multigrid scheme for the low-wing transport case. 


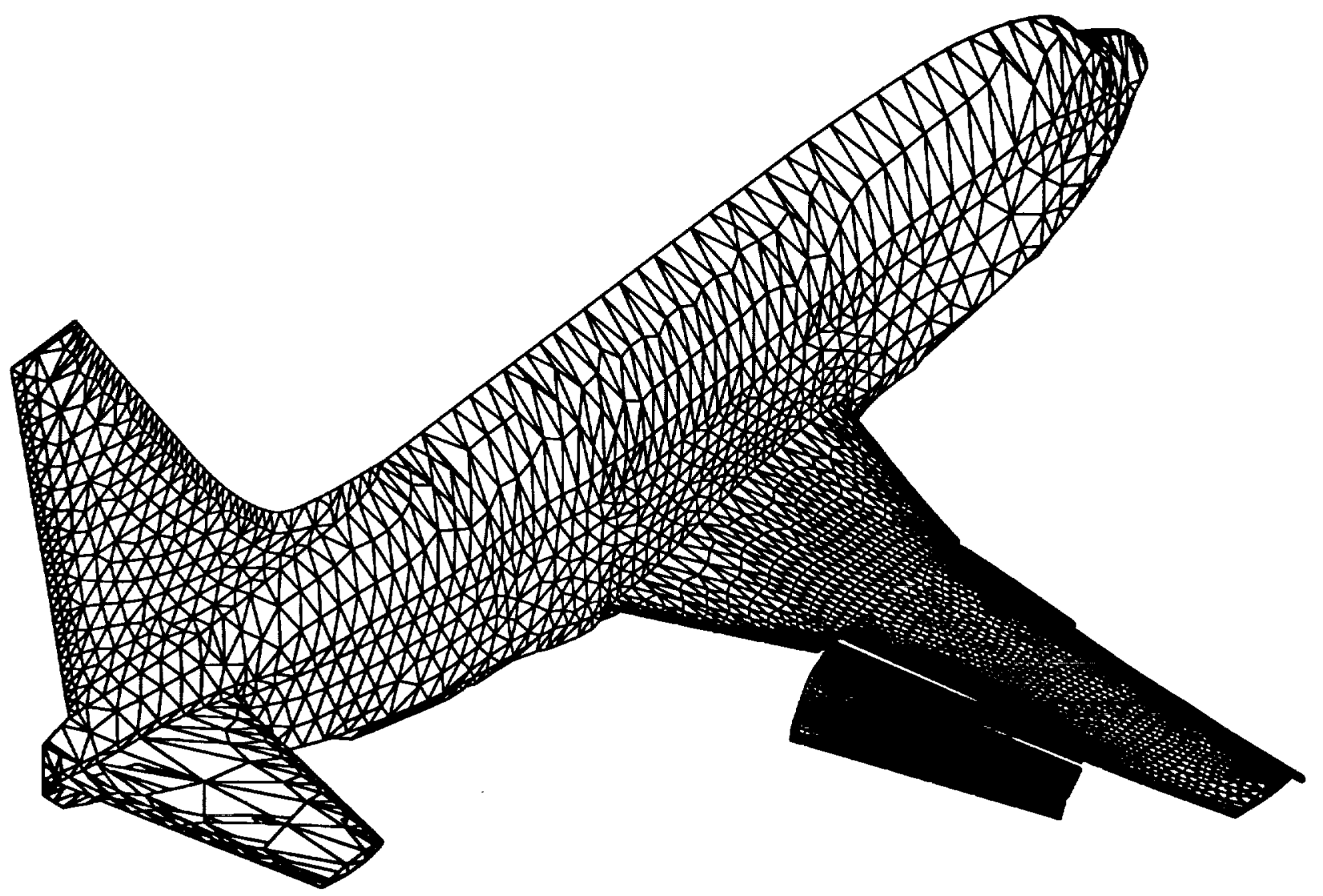

Figure 15: Surface mesh for a grid with 134,539 vertices about a fuselage-wing-flap geometry. 


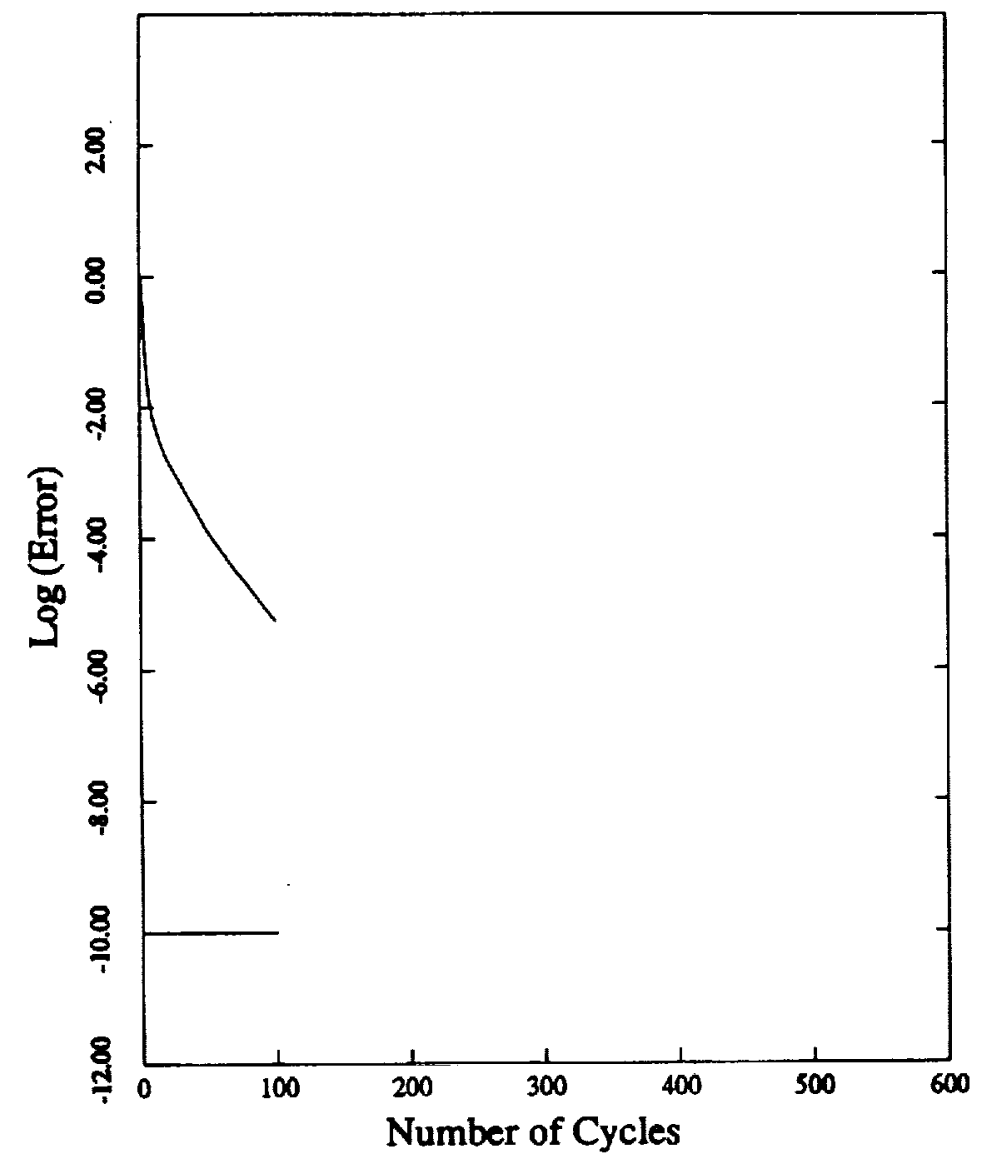

Nnode : 134539 Ncyct : 100 Ncycf : 0

Resid1 : 0.83E-01 Resid2 : 0.45E-06 Rate : 0.8847

Figure 16: Convergence history of the 6-level agglomerated multigrid scheme for the fuselagewing-flap geometry. 



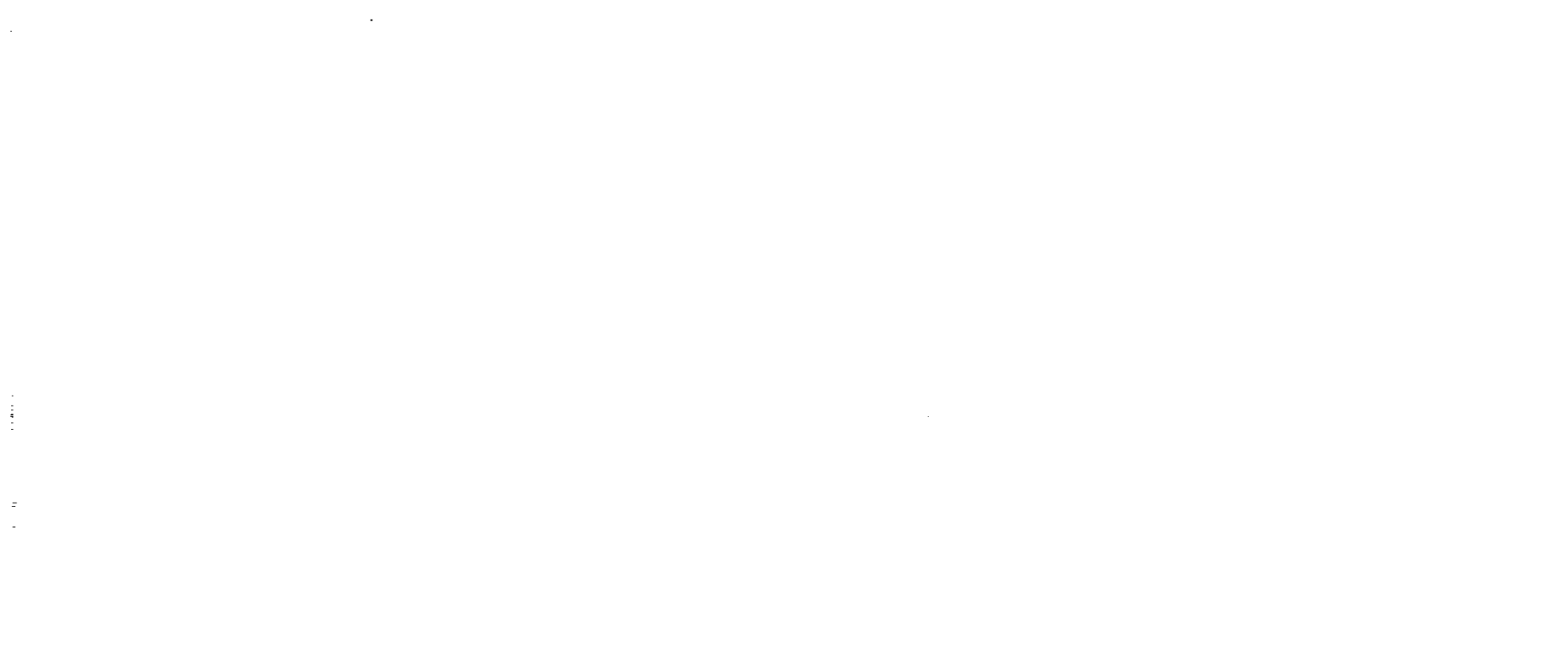


Public reporting burden for this callection of information is estimated to average 1 hour per response, including the time for reviewing instructions, searching existing data sources, athering and andeting and reviewing the collection of information. Send comments regarding this burden estimate or any ather aspect of this 8 athering and maintaining the data needed, and completing and revew. Washington Headquarters Services, Directorate for Information Operations and Reports, 1215 Jefferson Davis Highway, Suite 1204. Arlington. VA 22202-1302, and to the Office of Management and Budget, Paperwork Reduction Project (0704-0188), Washington. DC 20503.
1. AGENCY USE ONLY(Leave blank)
2. REPORT DATE
3. REPORT TYPE AND DATES COVERED
January 1994 Contractor Report

4. TITLE AND SUBTITLE AGGLOMERATION MULTIGRID FOR THE THREE-DIMENSIONAL EULER EQUATIONS

5. FUNDING NUMBERS

C NAS1-19480

WU 505-90-52-01

\section{AUTHOR(5)}

V. Venkatakrishnan

D. J. Mavriplis

7. PERFORMING ORGANIZATION NAME(S) AND ADDRESS(ES)

Institute for Computer Applications in Science

and Engineering

Mail Stop 132C, NASA Langley Research Center

Hampton, VA 23681-0001

9. SPONSORING/MONITORING AGENCY NAME(S) AND ADDRESS(ES)

National Aeronautics and Space Administration

Langley Research Center

Hampton, VA 23681-0001

8. PERFORMING ORGANIZATION

REPORT NUMBER

ICASE Report No. 94-5

\section{SUPPLEMENTARY NOTES}

Langley Technical Monitor: Michael F. Card

Final Report

Submitted to AIAA Journal

12a. DISTRIBUTION/AVAILABILITY STATEMENT

12b. DISTRIBUTION CODE

Unclassified-Unlimited

Subject Category 64,02

13. ABSTRACT (Maximum 200 words)

A multigrid procedure that makes use of coarse grids generated by the agglomeration of control volumes is advocated as a practical approach for solving the three-dimensional Euler equations on unstructured grids about complex configurations. It is shown that the agglomeration procedure can be tailored to achieve certain coarse grid properties such as the sizes of the coarse grids and aspect ratios of the coarse grid cells. The agglomeration is done as a preprocessing step and runs in linear time. The implications for multigrid of using arbitrary polyhedral coarse grids are discussed. The agglomeration multigrid technique compares very favorably with existing multigrid procedures both in terms of convergence rates and elapsed times. The main advantage of the present approach is the ease with which coarse grids of any desired degree of coarseness may be generated in three dimensions, without being constrained by considerations of geometry. Inviscid flows over a variety of complex configurations are computed using the agglomeration multigrid strategy.

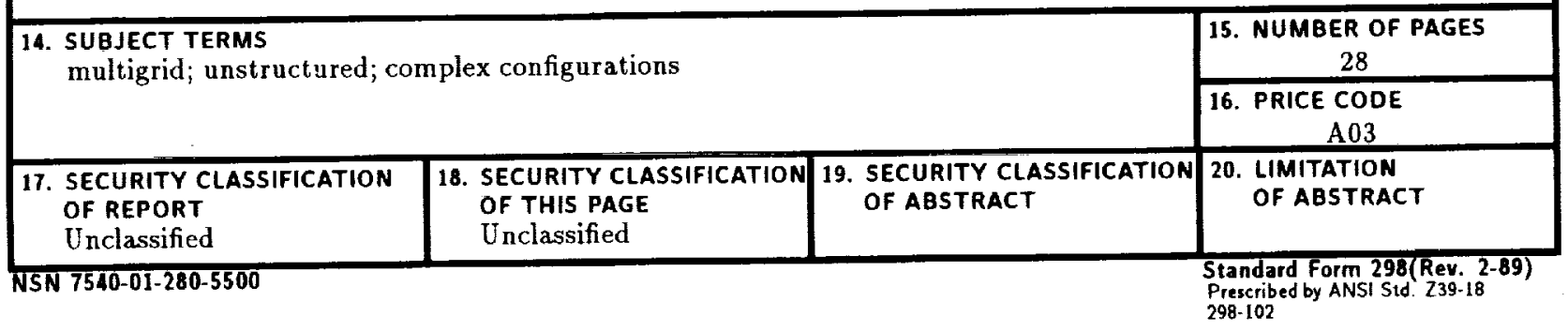

